\title{
COLD NEUTRON SCATTERING STUDIES OF FRUSTRATED PYROCHLORE ANTIFERROMAGNETS
}

\author{
J. S. GARDNER ${ }^{1, *}$, K. C. RULE ${ }^{2}$, J. P. C. RUFF², J. P. CLANCY², and B. D. GAULIN ${ }^{2}$ \\ ${ }^{1}$ Indiana University, Department of Physics \\ Bloomington, IN 47408 USA \\ ${ }^{1}$ National Institute of Standards \& Technology \\ Gaithersburg, MD 20899 USA \\ ${ }^{2}$ McMaster University, Department of Physics \\ Astronomy, Haminton, ON, L8S 4M1 Canada \\ *Corresponding author. E-mail : jason.gardner@nist.gov
}

Received December 31, 2010

In this paper we review the neutron scattering work performed on 3 different antiferromagnetic pyrochlores which reveal how the character of the magnetic interactions plays a major role on the eventual outcome of the magnetic ground state. $\mathrm{Tb}_{2} \mathrm{Ti}_{2} \mathrm{O}_{7}, \mathrm{Er}_{2} \mathrm{Ti}_{2} \mathrm{O}_{7}$ and $\mathrm{Y}_{2} \mathrm{Mo}_{2} \mathrm{O}_{7}$ have all been extensively studied over the past 15 years and are known to display, respectively, spin liquid, long range ordered and glassy ground states. Although detailed experiments have been performed on these compounds, and much is known about their low temperature properties, a detailed theoretical understanding of their ground states remains elusive.

KEYWORDS : Pyrochlore, Neutron Scattering, Frustration

\section{INTRODUCTION}

The combination of near-neighbour antiferromagnetic interactions and certain lattice symmetries, based on triangles or tetrahedra, lead to a phenomena known broadly as geometrical frustration. [1] Such systems are found in nature in a variety of cubic pyrochlore, spinel and Laves phase materials, where the magnetic moments can arise from either rare earth or transition metal electrons. Typically, the rare earth moments are relatively large and are only weakly coupled by exchange, such that the competition between antiferromagnetic exchange and dipolar interactions is important. Antiferromagnetic exchange interactions tend to be stronger, and therefore dominant in transition metal-based materials, and the manifestation of geometrical frustration is different. This review focusses on experimental progress in this area from the last ten years, looking at 3 samples with different magnetic species decorating the pyrochlore structure.

In a frustrated magnet, the spinsystem under question cannot simultaneously satisfy all of its near neighbour pairwiseinteractions and the resulting ground state can possess a macroscopic degeneracy. This situation is illustrated schematically in Fig. 1a and b. Figure 1a shows two antiferromagnetically-coupled spins on a triangle and the frustration which arises if their ground state configuration requires nearest neighbour spins to be antiparallel. The tetrahedron, shown in Fig. $1 \mathrm{~b}$ is to three dimensions what the triangle is to two dimensions, and a related degeneracy is relevant to finding a ground state arrangement for four near neighbour spins at the vertices of a tetrahedron.

Figure 1c shows the metallic ions in the unit cell of

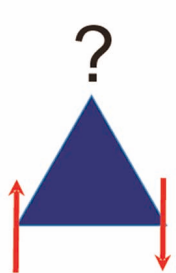

(A)

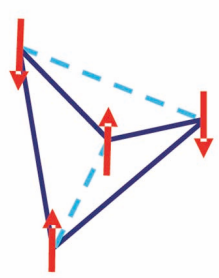

(B)

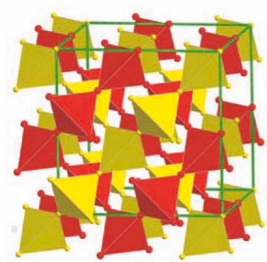

(C)
Fig. 1. Subunits of Geometrically Frustrated Magnets. (A) Two of Three Ising Spins Located on a Triangular Unit can form An Up-Down Pair, While the Spin at the Apex is Frustrated.

(B) Ising Spins on a Tetrahedron Showing Four Satisfied

(Antiferromagnetic)and Two Frustrated (Ferromagnetic) Bonds. (C)The Oxide Pyrochlore Unit Cell Showing the A (light) and B (Dark)Metal Sublattices, Each of Which Independently forms a Network of Corner Sharing Tetrahedra 
the cubic pyrochlore lattice with the general formula $\mathrm{A}_{2} \mathrm{~B}_{2} \mathrm{O}_{7}$. Both the $\mathrm{A}$ and $\mathrm{B}$ sites individually form a network of corner-sharing tetrahedra, and if either site is magnetic withpredominantly near-neighbour interactions a degree of frustration can exist.

Theoretical arguments have shown that both Ising and continuous (XY and Heisenberg) spin systems residing on a $3 \mathrm{D}$-pyrochlore lattice can possess a large and macroscopic degereracy when combined with certain anisotropies. [2-6] Local order, where the sum of the vector spins on a given tetrahedron is 0 (ie $\sum_{\mathrm{i}} \mathrm{S}_{\mathrm{i}}=0$ ) is often found in antiferromagnets, but only one sample is known to globally order in this configuration. [7] Villain, [4] coined the term co-operative paramagnet to describe the low temperature properties of these poorly correlated systems.

In this paper we will review neutron scattering results from three such pyrochlores, $\mathrm{Tb}_{2} \mathrm{Ti}_{2} \mathrm{O}_{7}, \mathrm{Er}_{2} \mathrm{Ti}_{2} \mathrm{O}_{7}$ and $\mathrm{Y}_{2} \mathrm{Mo}_{2} \mathrm{O}_{7}$. All three examples have only a single magnetic species, the first two have magnetic rare-earth ions decorating the A site of the pyrochlore lattice, while the third has a magnetic $4 \mathrm{~d}$ transition metal ion decorating the $\mathrm{B}$ site of the pyrochlore lattice. $\mathrm{The} \mathrm{Tb}^{3+}$ moments in $\mathrm{Tb}_{2} \mathrm{Ti}_{2} \mathrm{O}_{7}$ possesses an easy axis (Ising-like) anisotropy, while $\mathrm{Er}^{3+}$ in $\mathrm{Er}_{2} \mathrm{Ti}_{2} \mathrm{O}_{7}$ possesses planar (XY-type) anisotropy. The third example $\mathrm{Y}_{2} \mathrm{Mo}_{2} \mathrm{O}_{7}$ has orbitallyquenched $\mathrm{Mo}^{4+}$ moments, which are therefore isotropic (Heisenberg-like).

The local chemical environment around the pyrochlore $A$ and $B$ sites is not the same, with the $A$ site being eightfold coordinated by a distorted cube of $\mathrm{O}^{2-}$ ions, while the $\mathrm{B}$ site is coordinated within $\mathrm{BO}_{6}$ octahedra. [8] This local structure can profoundly influence the magnetism in these compounds, particularly for the rare earth moment at the A site. The nature of the distortion of the oxygen environment determines the crystalline electric field splittings relevant to the rare earth ion, which in turn determine the nature of the rare earth magnetic moment and its anisotropies. The transition metal moment tends to be spin only, due to quenching of its orbital magnetic moment.

To establish the fundamental magnetic properties of new materials,basic structural characterization (typically x-ray diffraction), susceptibility, magnetization and heat capacity measurements are usually required. In metallic materials an understanding of the transport properties and Fermi surface properties are also required. To make further progress, microscopic probes of magnetism must be employed, including NMR, $\mu$ SR and neutron scattering.

Of these experimental probes, neutron scattering is the only one capable of providing information on the static and dynamicbehaviour of the magnetic system as a function of wavevectorspanning the entire Brillouin zone, and as a function of frequencycovering spin dynamics on time scales from $10^{-7}$ seconds to $10^{-13}$ seconds. For this reason, neutron scattering is oftenregarded as an essential probe in the understanding of magneticsystems.

The neutron is, of course, electrically neutral, but carries a spin $1 / 2$ magnetic moment. As such neutron scattering is a weak probe of matter, inthe sense that neutrons penetrate deeply into most materials and largevolumes of material are often needed to carry out neutron experiments,especially inelastic neutron scattering experiments. It is also a weakprobe of matter in the sense that the scattering cross section can be described within the Born Approximation and Fermi's Golden Rule. This is asignificant advantage as it means that theoretical calculations can becompared directly to the relevant neutron scattering measurements.

The double differential scattering cross section describing the relative magnetic neutron scattering into an element of solid angle, $\mathrm{d} \Omega$, and with the scattered neutron having a final energy between $E_{f}$ and $E_{f}+d E_{f}$ can be writtenas

$$
\frac{d^{2} \sigma}{d \Omega_{f} d E_{f}}=\frac{N}{\hbar} \frac{k_{f}}{k_{i}} p^{2} e^{-2 W} \Sigma_{\alpha \beta}\left(\delta_{\alpha, \beta}-\hat{Q}_{\alpha} \hat{Q}_{\beta}\right) S^{\alpha \beta}(\mathbf{Q}, \omega)
$$

Here $\mathrm{k}_{\mathrm{i}}$ and $\mathrm{k}_{\mathrm{f}}$ are the initial and scattered neutron wavevectors; $\mathrm{p}=\frac{1}{2} \gamma \mathrm{r}_{\mathrm{o}} g f(\mathbf{Q})$ where $\gamma$ is the gyromagnetic ratio of the neutron, $r_{o}$ is the classical electron radius, $g$ is the Lande g-factor, and $f(\mathbf{Q})$ is the magnetic form factor appropriate to the magnetic moment density of the atom; and $\mathrm{e}^{-2 \mathrm{~W}}$ is the Debye-Waller factor. The physics associated with the magnetic moments is contained in:

$$
S^{\alpha \beta}(\mathbf{Q}, \omega)=\frac{1}{2 \pi} \int_{-\infty}^{\infty} d t e^{-i \omega t} \Sigma_{l} e^{\left(i \mathbf{Q} \cdot \mathbf{r}_{1}\right)}\left\langle S_{0}^{\alpha} S_{l}^{\beta}(t)\right\rangle
$$

Integrating over all energy and over a complete Brillouin zone gives rise to the sum rule:

$$
\int_{-\infty}^{\infty} d \omega \int_{B Z} d \mathbf{Q} S^{\alpha \beta}(\mathbf{Q}, \omega) \sim S(S+1) \delta_{\alpha \beta}
$$

This relation implies that the total magnetic scattering scales as the size of the moment with $\mathrm{S}(\mathrm{S}+1)$, and that systems with suppressed magnetic ordering and therefore suppressed ordered magnetic moments necessarily exhibit enhanced inelastic scattering so as to satisfy the above sum rule. Excellent comprehensive reviews of neutron scattering exist and are referenced for the interested reader. [9]

The high temperature uniform susceptibility of $\mathrm{Tb}_{2} \mathrm{Ti}_{2} \mathrm{O}_{7}, \mathrm{Er}_{2} \mathrm{Ti}_{2} \mathrm{O}_{7}$ and $\mathrm{Y}_{2} \mathrm{Mo}_{2} \mathrm{O}_{7}$, are well described by a Curie-Weiss form, with $\theta \mathrm{cw} \sim-19 \mathrm{~K},-22 \mathrm{~K}$ and $-201 \mathrm{~K}$ respectively, indicating net antiferromagnetic correlations. 
The paramagnetic moment, also found from the CurieWeiss fits, are roughly consistent with the expected freeion value in the case of the rare earth pyrochlores $\mathrm{Tb}_{2} \mathrm{Ti}_{2} \mathrm{O}_{7}$ and $\mathrm{Er}_{2} \mathrm{Ti}_{2} \mathrm{O}_{7}$. The ground states these three pyrochlore antiferromagnets display are varied: at low temperatures $\mathrm{Tb}_{2} \mathrm{Ti}_{2} \mathrm{O}_{7}$ has a spin liquid ground state, [10] $\mathrm{Er}_{2} \mathrm{Ti}_{2} \mathrm{O}_{7}$ orders at $\sim 1.1 \mathrm{~K}[11]$ and $\mathrm{Y}_{2} \mathrm{Mo}_{2} \mathrm{O}_{7}$ freezes into a glass state at $22.5 \mathrm{~K}$. [12]

The low temperature diffuse scattering, associated with short range correlations is shown in figure 2 . The
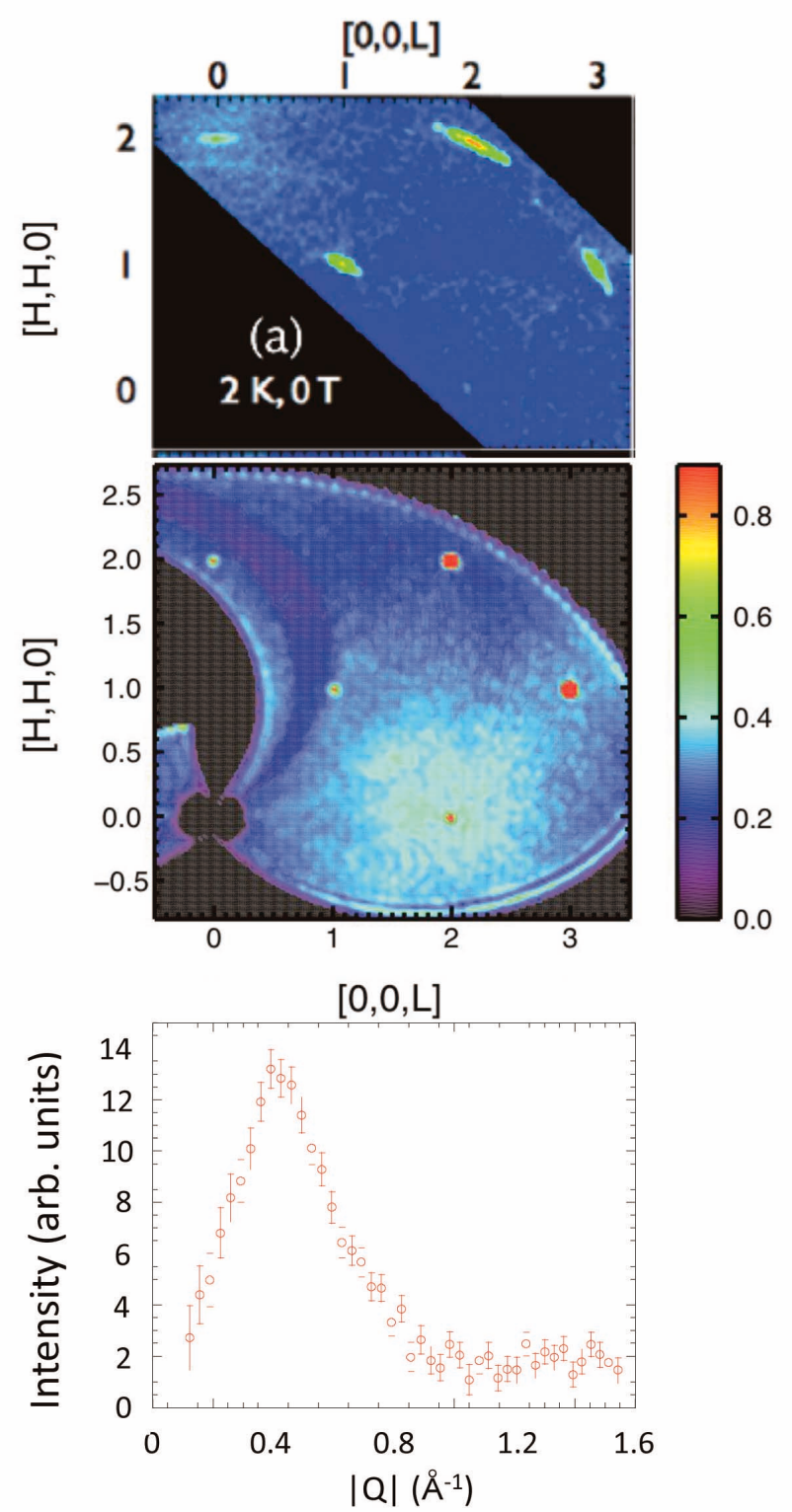

Fig. 2. Elastic Neutron Scattering of the 3 Samples at $\sim 1 K$. The Paramagnetic State of the Ordered Pyrochlore, $\mathrm{Er}_{2} \mathrm{Ti}_{2} \mathrm{O}_{7}$ Just Above the Ordering Temperature at $2 \mathrm{~K}$ (top) [13]. The Scattering from the Cooperative Paramagnet, $\mathrm{Tb}_{2} \mathrm{Ti}_{2} \mathrm{O}_{7}$ at $1 \mathrm{~K}$ (middle)[14], and Polycrystalline Results from the Spin Glass, $\mathrm{Y}_{2} \mathrm{Mo}_{2} \mathrm{O}_{7}$ at $1.4 \mathrm{~K}$. [15] four dimensional dynamic structure factor $S(\mathbf{Q}, \hbar \omega)$ can be studied using neutron scattering techniques, due to the availibility of large crystals often grown using floating zone image furnace techniques. [16] The top panel shows a similar spectra from $\mathrm{Er}_{2} \mathrm{Ti}_{2} \mathrm{O}_{7}$ at $2 \mathrm{~K}$. [14] The middle panel shows the results from a similar sized, $9 \mathrm{~g}$, single crystals of $\mathrm{Tb}_{2} \mathrm{Ti}_{2} \mathrm{O}_{7}$ at $1 \mathrm{~K}$. $[13,17] \mathrm{The}$ lower panel shows the powder averaged elastic magnetic scattering from $\mathrm{Y}_{2} \mathrm{Mo}_{2} \mathrm{O}_{7}$ at $1.4 \mathrm{~K}$, obtained by subtracting a $50 \mathrm{~K}$ data set from a $1.5 \mathrm{~K}$ data set. [14] Although materials chemists and physicists are trying hard to grow single crystals of $\mathrm{Y}_{2} \mathrm{Mo}_{2} \mathrm{O}_{7}$, [18] they have thus far been unsuccessful and only polycrystalline samples are currently available. In all three of these samples, broad diffuse magnetic scattering is seen in Q-space, indicative of spin correlations on a length scale small compared to the dimensions of the unit cell $\sim 10.1 \AA$. This diffuse scattering continues to the lowest temperatures in the spin liquid $\mathrm{Tb}_{2} \mathrm{Ti}_{2} \mathrm{O}_{7}$, while it builds into Bragg peaks in $\mathrm{Er}_{2} \mathrm{Ti}_{2} \mathrm{O}_{7}$ as the system is lowered trough the Néel temperature. $[13,19,20]$ However, its clear from detailed scans through the Bragg peaks, that short range correlations persist well below the ordering temperature at $50 \mathrm{mK}$ (see fig. 3) and a true ordered, frozen state may never be achieved. Lago et al., [21] previously observed a large muon relaxation rate below the ordering temperature, consistent with dynamic short range spin correlations.

Inelastic neutron scattering from these 3 pyrochlore antiferromagnets reveal some of the major differences between their ground state properties. $\mathrm{Tb}_{2} \mathrm{Ti}_{2} \mathrm{O}_{7}$, a cooperative paramagnet or classical spin liquid, has quasielastic

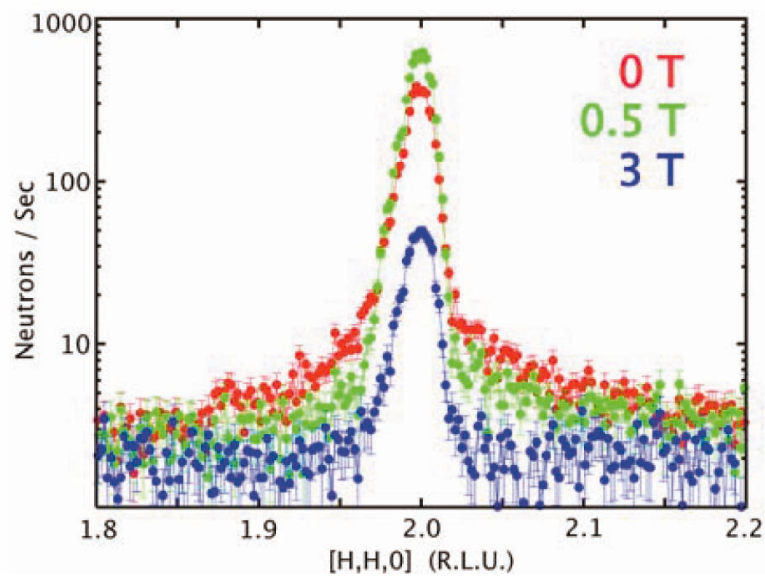

Fig. 3. The Elastic Scattering Around the (2 20$)$ Position in Reciprocal Space in $\mathrm{Er}_{2} \mathrm{Ti}_{2} \mathrm{O}_{7}$ at $50 \mathrm{mK}$ with An Applied Field in the $\left[\begin{array}{lll}1 & -1 & 0\end{array}\right]$ Direction. The 3T Data Set is a Good Measure of the Instrumental Background and Resolution. The Existence of a Broad, Diffuse Component to the Scattering in Zero Applied Magnetic Field is Clear, Indicating Short-Range Magnetic Correlations that Coexist with Long-Range Order in Zero Applied Field.[13] 
scattering and a low lying excitation at $\sim 1.5 \mathrm{meV}(0.36$ $\mathrm{THz}$ ) which displays some dispersion, and therfore some degree of spin-spin correlations, with a partial softening at $\mathbf{Q}$ 's where $\mathbf{S}(\mathbf{Q})$ is a maxima, see figure 4 . A simple model involving near-neighbor spin correlations on the
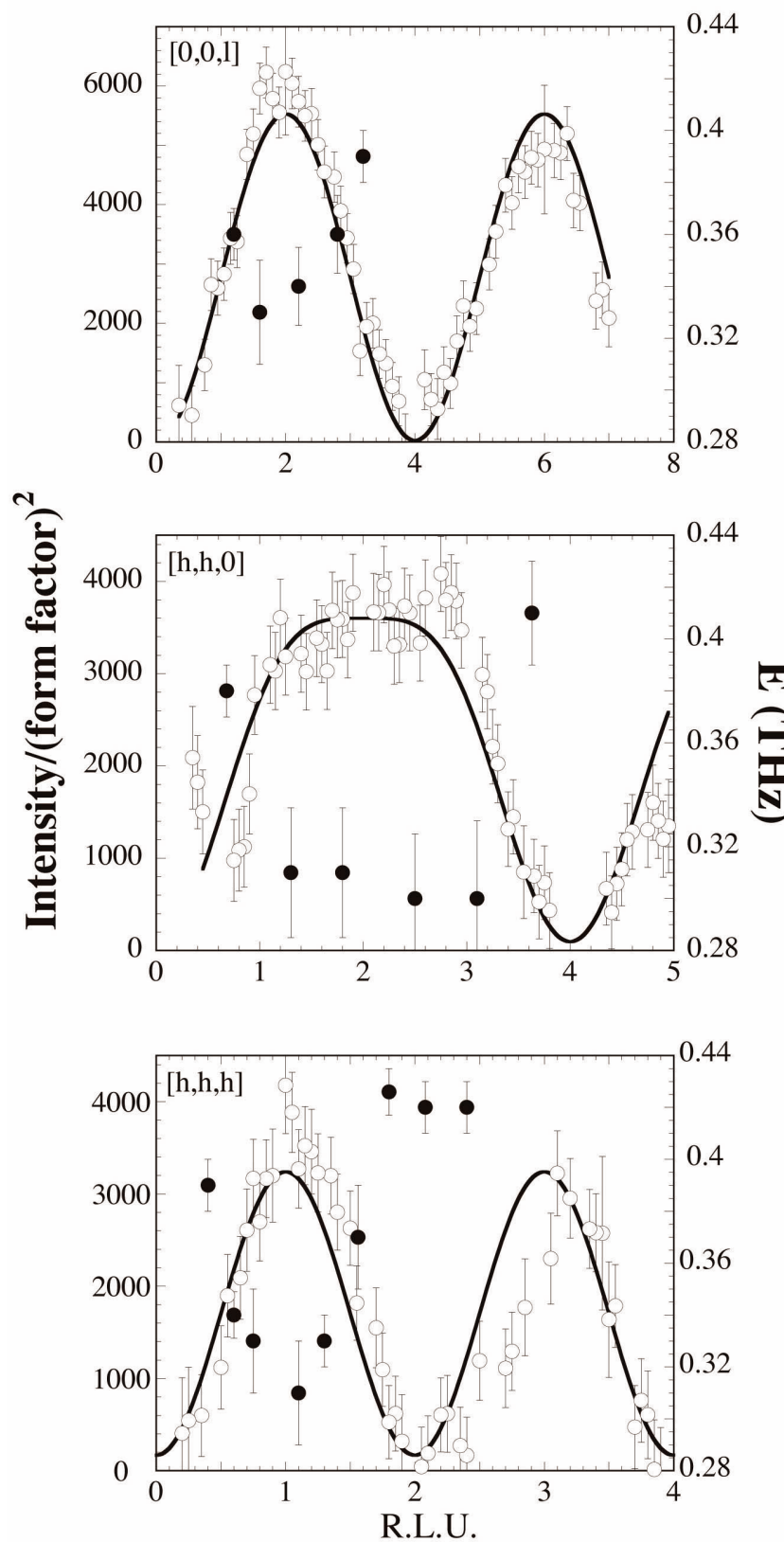

Fig. 4. Line Plots Through the Checkerboard Board Pattern of Diffuse Magnetic Scattering (Open Symbols) along High SymmetryDirections of the Cubic Lattice in $\mathrm{Tb}_{2} \mathrm{Ti}_{2} \mathrm{O}_{7}$. To Complement These Data, The Dispersion of the Lowest Lying Magnetic Excitation, at $4 \mathrm{~K}$, Isalso Plotted (Closed Symbols), and Shows that Minima in the Spin Excitation Dispersion Coincide with Peaks in the Diffuse Magnetic Scattering. Fits to the Diffuse Scatteringare Shown and Discussed in the Text. (22) pyrochlore lattice alone results on a structure factor of

$$
\begin{aligned}
S(\mathbf{Q})= & 1+a\{\cos (h \pi / 2) \cos (k \pi / 2)+\cos (h \pi / 2 \cos (l \pi / 2) \\
& +\cos (h \pi / 2 \cos (l \pi / 2)+\cos (k \pi / 2) \cos (l \pi / 2)\} .
\end{aligned}
$$

where $\mathrm{a}$ is the nearest-neighbor spin-spin correlation function, which has the value $-1 / 3$ at $\mathrm{T}=0$, and $h, k$ and $l$ are the Miller indices defining a place in reciprocal space. As shown in Figure 4, this model of the least correlated spin system imaginable, describes the data reasonably well in the three high symetry directions.

The spin dynamics in the spin glass, $\mathrm{Y}_{2} \mathrm{Mo}_{2} \mathrm{O}_{7}$, are strongly suppressed below the system freezing temperature of $20 \mathrm{~K}$. No other inelastic features have been seen below $5 \mathrm{meV}$ in the powder studies, see figure 5. At high temperatures, very broad quasielastic scattering dominates the low energy spectra. As the temperature is lowered below $40 \mathrm{~K}$, the spectral weight builds up around $\hbar \omega=0$. Below $20 \mathrm{~K}$, any low energy spectral weight is within the instrumental resolution. [15] Using neutron spin echo, a dynamical spin system is seen down to at least $10 \mathrm{~K}$. [23] Recent muon relaxation measurements have observed spin dynamics on the microsecond timescale at $50 \mathrm{mK}$. [21]
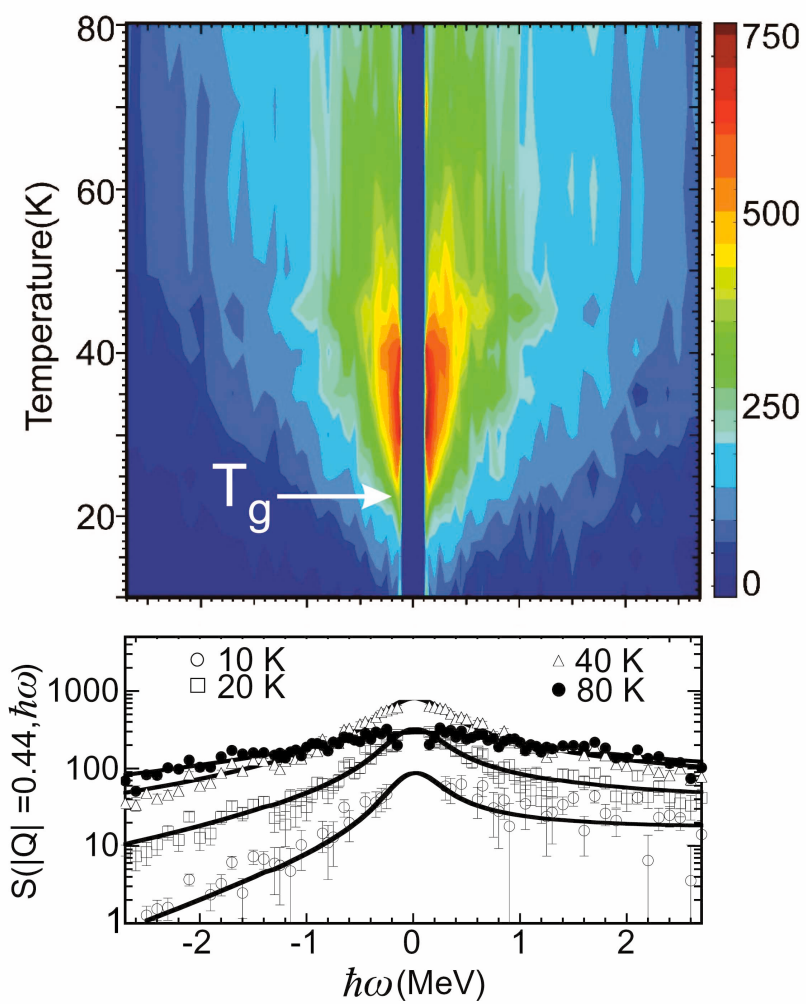

Fig. 5. Background-Subtracted and Normalized Inelasticscattering Data for $S(\mathbf{Q}=0.44 \AA \mathcal{\Lambda} \omega)$ at Temperatures between 10 and 80 K. (b) Representat Ive Energy Scans from Which the Contour Map was Made. [15] 
Collective dynamics or spin waves have been observed in $\mathrm{Er}_{2} \mathrm{Ti}_{2} \mathrm{O}_{7}$ below $1 \mathrm{~K}$. At $2 \mathrm{~K}$ in the paramagnetic state, significant spectral weight is observed below $1 / 2 \mathrm{meV}$ at specific Bragg positions, for example the (2 20 ) (see fig. 6). As the temperature is lowered, this scattering builds up and forms spin waves emanating from Bragg positions with a linear dispersion, as expected for an ordered antiferromagnet. These studies have been developed further in an applied field along the $1-10$ and it was observed that these bulk excitations are supressed in $1 \mathrm{~T}$, by quantum fluctuations and $\mathrm{Er}_{2} \mathrm{Ti}_{2} \mathrm{O}_{7}$ enters a new quantum paramagnetic state on passing through a magnetic field induced quantum critical point. [13]

One can appreciate the complexity of the magnetism in rare-earth $\mathrm{A}_{2} \mathrm{~B}_{2} \mathrm{O}_{7}$ pyrochlores. After several decades of study, it is clear that for the magnetic A-site pyrochlore samples the local crystalline environment, or crystal field Hamiltonian is very important. Specifically, the splitting of the $2 \mathrm{~J}+1$ degenerate ground state of the rare earth ions determaines wheter the system has an easy plane or an easy axis with respectto the quantization axis which is $<111>$ for pyrochlores. The magnetic B-site pyrochlores are not as well studied. There has been some recent studies on unexpected transport properties in metallic and nominally non-magnetic pyrochlores, for example, $\mathrm{Cd}_{2} \mathrm{Re}_{2} \mathrm{O}_{7}$ [24] which is a superconductor at sufficiently low temperatures. Finally, when both the A and B sites are magnetic, complex and delicately balanced interactions can result in some unusual properties, such as the spontaneous Hall effect seen in $\mathrm{Pr}_{2} \mathrm{Ir}_{2} \mathrm{O}_{7}$ [25] without magnetic dipole ordering as well as the complex magnetism seen in $\mathrm{Tb}_{2} \mathrm{Mo}_{2} \mathrm{O}_{7}$. [26,27]

Hopefully in this short review one has gained an appreciation for the diverse phenomena displayed by frustrated pyrochlore antiferromagnets. Not only does

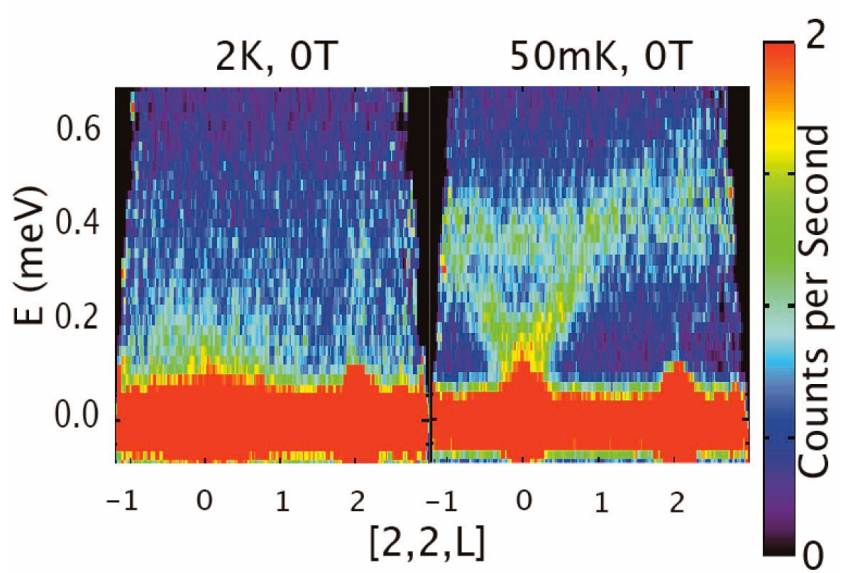

Fig. 6. Inelastic Neutron Scattering Spectra in $\mathrm{Er}_{2} \mathrm{Ti}_{2} \mathrm{O}_{7}$. Data at $\mathrm{T}=2 \mathrm{~K}$ Shows the System in its Paramagnetic State with Significant Quasielastic Scattering. At $50 \mathrm{mK}$, in the Ordered State, the Dispersion of Spin Waves is Shown along the [2 2 L] Line in Reciprocal Space, Joining the 220 and 222 Wave Vectors. [13] near neighbour exchange play a crucial role but dipolar interactions and the nature of the spin anisotropy can significantly alter the magnetic ground state in these magnets, due to the delicate balance from which the ground state arises. For a more indepth review of frustrated pyrochlore magnets, both antiferromagnets and ferromagnets, the authors direct your attention to a recent Review of Modern Physics. [28]

\section{REFERENCES}

[ 1 ] See, for example, Frustrated Spin Systems, edited by H. T.Diep (World Scientific, Singapore, 2004).

[2] P. W. Anderson, Phys. Rev., 102, 1008 (1956).

[3 ] J. N. Reimers, Phys. Rev. B., 45, 7287 (1992)

[4] J. Villain, Z. Phys. B. 33, 31 (1979).

[ 5 ] R. Moessner and J. T. Chalker, Phys. Rev. B 58, 12049 (1998).

[6 ] R. Moessner and J. T. Chalker, Phys. Rev. Lett. 80,2929 (1998).

[7 ] G. Ferey, R. de Pape, M. Leblanc and J. Pannetier, Rev. Chim. Miner, 23, 474 (1986).

[ 8 ] M.A. Subramanian, G. Aravamudan, and G.V. Subba Rao, Prog. Solid State Chem., 15, 55 (1983).

[9] G. Shirane, S.M. Shapiro, and J.M. Tranquada,Neutron Scattering with a Triple Axis Spectrometer, (Cambridge UniversityPress, 2002).

[10] J.S. Gardner, S.R. Dunsiger, B.D. Gaulin, M.J.P. Gingras, J.E. Greedan, R.F. Kiefl, M.D. Lumsden, W.A. MacFarlane, N.P. Raju, J.E. Sonier, I. Swainson and Z. Tun, Phys. Rev. Lett., 82, 1012 (1999).

[11] J.D.M. Champion, M.J. Harris, P.C.W. Holdsworth, A.S. Wills, G. Balakrishnan, S.T. Bramwell, E. Cizmar, T. Fennell, J.S. Gardner, J. Lago, D.F. McMorrow, M. Orendac, A. Orendacova, D. McK. Paul, R.I. Smith, M.T.F. Telling and A. Wildes, Phys. Rev. B, 68, 020401 (R) (2003).

[12] M.J.P. Gingras, C.V. Stager, N.P. Raju, B.D. Gaulin, J.E. Greedan, Phys. Rev. Lett., 78, 947 (1997).

[13] J.P.C. Ruff,J.P. Clancy, A. Bourque, M.A. White, M. Ramazanoglu, J.S. Gardner, Y. Qiu, J.R.D. Copley, M.B. Johnson, H.A. Dabkowskaand B.D. Gaulin, Phys. Rev. Lett., 101, 147205 (2008).

[14] K.C. Rule, J.P.C. Ruff, B.D. Gaulin, S.R. Dunsiger, J.S. Gardner, J.P. Clancy, M.J. Lewis, H.A. Dabkowska, I. Mirebeau, P. Manuel, Y. Qiuand J.R.D. Copley, Phys. Rev Lett., 96, 177201 (2006).

[15] J.S. Gardner, B.D. Gaulin, S.-H. Lee, C. Broholm, N.P. Raju and J. E. Greedan, Phys. Rev. Lett., 83, 211 (1999).

[16] J.S. Gardner, B.D. Gaulin and D.McK. Paul, J. Crystal Growth, 191, 740 (1998).

[17] J. S. Gardner,A. Keren,G. Ehlers,C. Stock,Eva Segal,J. M. Roper,B. Fak,M. B. Stone,P. R. Hammar,D. H. Reichand B. D. Gaulin, Phys. Rev. B., 68, 180401(R) (2003).

[18] Private communication C.R. Wiebe and H. Zhou, Florida state University (2008).

[19] A. Poole, A.S. Wills and E. Lelievre-Berna, J. Phys.: Condens. Matter, 19, 452201 (2007).

[20] H.B. Cao, I. Mirebeau, A. Gukasov, P. Bonville and C. Decorse, Phys. Rev. B., 82, 104431 (2010).

[21] J. Lago, T. Lancaster, S.J. Blundell, S.T. Bramwell, F.L. Pratt, M. Shirai, and C. Baines, J. Phys.: Condens. Matter, 
17, 979 (2005).

[22] J.S. Gardner, B.D. Gaulin, A.J. Berlinsky, P. Waldron, S.R. Dunsiger, N.P. Raju, and J.E. Greedan, Phys. Rev. B 64, 224416 (2001).

[23] J.S. Gardner, G Ehlers, R.H. Heffner and F. Mezei, J. Mag. Mag. Mat. 226460 (2001).

[24] M.D. Lumsden, S.R. Dunsiger, J.E. Sonier, R.I. Miller, R.F. Kiefl, R. Jin, J. He, D. Mandrus, S.T. Bramwell and J.S. Gardner, Phys. Rev. Lett., 89, 147002 (2002).
[25] Y. Machida, S. Nakatsuji, S. Onoda, T. Tayama and T Sakakibara, Nature, 463210 (2010).

[26] B.D. Gaulin, J.N. Reimers, T.E. Mason, J.E. Greedan, and Z. Tun, Phys. Rev. Lett., 69, 3244, (1992).

[27] D.K. Singh, J.S. Helton, S. Chu, T.H. Han, C.J. Bonnoit, S. Chang, H.J. Kang, J.W. Lynn and Y.S. Lee, Phys. Rev. B 78, 220405 (2008).

[28] J. S. Gardner, M. J. P. Gingras, and J. E. Greedan, Rev. Mod. Phys., 82, 53 (2010). 